\title{
II Taller de Naturalezas Híbridas. Los límites ecológicos de lo urbano
}

\author{
Santiago-Gómez, Elvira'; Cortés-Vázquez, Jose A.; Diz, Carlos ${ }^{3}$ \\ ${ }^{1}$ Universidade da Coruña, Facultade Socioloxía, 0000-0003-4635-144X \\ 2Universidade da Coruña, Facultade Socioloxía, 0000-0003-3566-7924 \\ ${ }^{3}$ Universidade da Coruña, Facultade Socioloxía, 0000-0002-9753-7730
}

\section{RESUMEN}

La actividad "Il Taller de Naturalezas Híbridas. Los límites ecológicos de lo urbano", organizada por el Grupo de Innovación Docente ConCiencia Social y dirigida al alumnado de cuarto curso del Grado en Sociología, tuvo como objetivo ahondar en la práctica de formas de aprendizaje activo que permitan abordar fuera del aula problemas complejos, como lo son la gestión de residuos, la contaminación y las repercusiones para la naturaleza que se desprenden del modo de vida en la ciudad. La actividad consistió en visitar lugares estratégicos, emplazados en el ámbito rural y que dan servicio al ámbito urbano, explorando su uso, sus límites y su función de forma colectiva, creando un espacio colaborativo en el que co-producir reflexiones, experiencias, interpretaciones y saberes sobre los límites ecológicos de lo urbano, poniendo especial atención en la construcción social de estos espacios y en la función del mundo académico en su cuestionamiento crítico. Esta reflexión supera la clásica división entre lo urbano y lo natural como dominios separados, situándonos ante realidades híbridas, que retan las divisiones del conocimiento experto entre disciplinas aplicadas.

PALABRAS CLAVE: Naturaleza, Contaminación, Residuos, Urbano, Conciencia Ambiental

\section{CITA RECOMENDADA:}

Santiago-Gómez, Elvira; Cortés-Vázquez, Jose A.; Diz, Carlos (2020): II Taller de Naturalezas Híbridas. Los límites ecológicos de lo urbano. En De la Torre Fernández, E. (ed.) (2020). Contextos universitarios 
transformadores: Boas prácticas no marco dos GID. IV Xornadas de Innovación Docente. Cufie. Universidade da Coruña. A Coruña (págs. 373-382).

DOl capítulo: https://doi.org/10.17979/spudc.9788497497756.373

DOI libro: https://doi.org/10.17979/spudc.9788497497756

\section{ABSTRACT}

The activity "II Hybrid Nature Workshop. The ecological limits of the urban", organised by the ConCiencia Social Innovation Group and aimed at students in the fourth year of the Degree in Sociology, was focused on practice of forms of active learning that allow complex problems to be tackled outside the classroom, such as waste management, pollution and repercussions for nature that arise from the way of life in the cities. The activity consisted of visiting strategic places located in rural areas that serve the urban environment, exploring their use, limits and function collectively, creating a collaborative space in which to co-produce reflections, experiences, interpretations and knowledge about the ecological limits of the urban, while paying special attention to the social construction of these spaces and the role of the academic world in its critical questioning. This reflection overcomes the classic division between the urban and the natural as separate domains, placing us before hybrid entities that challenge the divisions of expert knowledge between applied disciplines.

KEY WORDS: Nature, Pollution, Waste, Urban, Environmental Awareness 


\section{INTRODUCCIÓN}

Tras los buenos resultados alcanzados con el I Taller de Naturalezas Híbridas celebrado en octubre de 2018, en el que se exploraron las "naturalezas urbanas" de A Coruña (Diz et al., 2019), la actividad "Il Taller de Naturalezas Híbridas. Los límites ecológicos de lo urbano" organizada por el Grupo de Innovación Docente ConCiencia Social y celebrada el 4 de octubre de 2019 estuvo dirigida al alumnado de cuarto curso del grado en Sociología. La actividad, de carácter optativo, tuvo como objetivo fundamental ahondar en la puesta en práctica de formas de aprendizaje activo que permitan abordar fuera del aula problemas complejos relacionados con la sostenibilidad ambiental, como lo son la gestión de residuos, la contaminación y las repercusiones para la naturaleza que se desprenden del modo de vida en la ciudad.

La capacidad de las universidades para afrontar los retos de la globalización y de la sociedad del conocimiento a partir de la innovación las convierte en instituciones de referencia en cuanto al impacto en el desarrollo sostenible de las actividades de docencia, investigación y gestión (Vallerys, 2006; Lozano et al., 2006). El informe final de la conferencia de Tibilisi del año 1977 ya establecía que las universidades, en su calidad de centros de investigación, de enseñanza y de formación de personal cualificado, deberían dar cada vez mayor cabida a la investigación sobre educación ambiental en sus currículos. El esfuerzo realizado por las instituciones educativas a la hora de incorporar los valores ambientales al sistema educativo fue reconocido por la ONU al declarar la década 2005-2014 como la Década de la Educación para el Desarrollo Sostenible. En esta dirección, el Grupo de Evaluación de la Sostenibilidad Universitaria de la CRUE (2011) define un conjunto de indicadores que permiten medir la contribución a la sostenibilidad de las iniciativas llevadas a cabo por las universidades, destacando las actuaciones relacionadas con la sensibilización ambiental, la gestión de los residuos y la introducción de contenidos y competencias relacionados con la sostenibilidad en el currículo y la docencia.

La preocupación por la sostenibilidad aparece recogida en el Plan de Acción 2013-2020 de la Universidade da Coruña, en el que se detalla que el elemento central de la responsabilidad 
social de la institución es su aspiración a convertirse en motor de cambio social a partir de un compromiso activo con su entorno más próximo, siguiendo un modelo de universidad responsable con su impacto ambiental. En línea con este propósito, en la UDC se crea el Campus Sustenta, un campus de especialización que promueve la excelencia en el conocimiento para la transición a estilos de vida sostenibles que protejan los recursos naturales, frenen el cambio climático y promuevan el bienestar.

A pesar de los avances realizados en los últimos años, el fortalecimiento de la conciencia ambiental y la introducción de la sostenibilidad en el currículo académico siguen siendo un reto importante para las instituciones de enseñanza superior (Savelyeva y McKenna, 2011). En este contexto, la Responsabilidad Social Universitaria (RSU) orientada a la sostenibilidad, encuentra estímulos en los acuerdos internacionales en materia ambiental, pero también barreras que dificultan la institucionalización del desarrollo sostenible en la rutina de la actividad universitaria. Estas dificultades están relacionadas con la cultura y la estructura de las universidades, la falta de colaboración entre personal docente e investigador y personal de administración y servicios o la falta de tiempo y de recursos (Abadía et al., 2012).

A la hora de enfrentarse a estas barreras, el grupo de Innovación Docente ConCiencia Social propone los siguientes objetivos: en el ámbito de la docencia busca aumentar el número de materias del Grado en Sociología que incluyan competencias relacionadas con la ciudadanía y los recursos naturales, manteniendo un compromiso activo con el fomento de las sinergias y las enseñanzas relacionadas con la sensibilización ambiental y la educación para la sostenibilidad. En el ámbito de las relaciones con el entorno se contempla la colaboración con organizaciones no gubernamentales, entidades públicas y empresas privadas. Por último, en el ámbito de la Universidad se establecen dos ejes de intervención: la adhesión al programa Green Campus y el fortalecimiento del Plan de Acción Tutorial. Los Talleres de Naturalezas Híbridas hasta ahora desarrollados tratan de avanzar en los tres ejes planteados incorporando la sostenibilidad al currículo académico, colaborando con las entidades del entorno y avanzando en la consecución del Plan de Acción Green Campus. Además, los talleres son una 
muestra de colaboración interdisciplinar entre docentes y de éxito en la organización colaborativa en un contexto de escasez de recursos.

\section{DESCRIPCIÓN DE LA EXPERIENCIA}

Los Talleres de Naturalezas Híbridas tienen como objetivo diseñar una herramienta de innovación docente replicable que permita desarrollar dinámicas docentes más simétricas basadas en la colaboración, la descentralización y la co-construcción del conocimiento (Diz et al., 2019). Se trata de reflexionar en torno a la relación entre sociedad, naturaleza y ciudad desde un acercamiento multidisciplinar y aplicado, basado en los procesos de enseñanzaaprendizaje saludables y sostenibles, estableciendo una reflexión abierta entre profesorado, personal experto y alumnado. Metodológicamente, el taller propone una triangulación entre la etnometodología, la etnografía y la Investigación Acción Participativa. Los talleres mantienen una aproximación interdisciplinar entre las Ciencias Sociales y las Ciencias Medioambientales hacia el conflicto en torno al uso y la gestión del territorio que permita comprender y analizar la relación entre naturaleza, sociedad y ciudad y los límites del estilo de vida urbano, y suponen un apoyo a los contenidos de las materias optativas del Grado en Sociología: "Riesgo, cultura y medioambiente"; "Globalización, Medio Ambiente y Población" y "Acción Colectiva y Cooperación Social".

En la primera materialización de esta propuesta, que tuvo lugar en 2018 bajo el título "I Taller de Naturalezas Híbridas", la atención se dirigió hacia las naturalezas urbanas como concepto y campo de estudio a partir del que problematizar los límites y fronteras de conocimiento dentro de la sociología. El alumnado pudo analizar, en contacto directo con la realidad estudiada, la relación entre naturaleza, sociedad y ciudad a través de espacios urbanos liminales y periféricos, donde lo natural, lo social y lo urbano se encuentran entrelazados e hibridados, formando un ensamblaje territorial densamente complejo y escasamente estudiado. Para ello la jornada se estructuró en un debate-ruta-debate, primero asistiendo a una conferencia en la facultad y visitando a continuación varios espacios híbridos de A Coruña (parques, lagunas, 
cuencas de ríos, corredores verdes, etc.). En esta actividad se contó con la colaboración del arquitecto y urbanista lago Carro, miembro de la Asociación Cultural Ergosfera y de Partidarios da Cidade Sociedade Cooperativa Galega.

El éxito de la primera edición animó al Grupo de Innovación Docente a organizar una segunda edición en la que se mantuviese el foco de atención en la conciencia ambiental, la responsabilidad social, los procesos de hibridación y el aprendizaje activo y sensorial, pero revisando el lugar de la visita y el material de aprendizaje previo y reforzando el anclaje del contenido de las materias optativas del último curso del grado.

El "Il Taller de Naturalezas Híbridas. Los límites ecológicos de lo urbano", consistió en una jornada que se inició otra vez con un debate en la facultad de sociología, para el que se contó con la participación de Leandro del Río en representación de la plataforma ciudadana "Salvemos Ordes", seguido de una salida con destino a la central Térmica de CercedaMeirama y al Vertedero de SOGAMA. La actividad, que en esta ocasión desplazaba su objeto de atención de las fronteras internas de la ciudad a sus fronteras externas, desde las cuales interrogar los límites ecológicos de lo urbano, contó con una ayuda del Departamento de Sociología y Ciencias de la Comunicación de 250 euros - cantidad que fue dedicada al alquiler de un autobús- y consistió fundamentalmente en debatir/visitar estos lugares emplazados en el ámbito rural para dar servicio al ámbito urbano, explorando su uso y función de forma colectiva, creando un espacio colaborativo en el que co-producir reflexiones, experiencias, interpretaciones y saberes sobre los límites ecológicos de lo urbano, poniendo especial atención en la construcción social y simbólica de estos espacios y en la función inexcusable del mundo académico en su estudio y cuestionamiento crítico. 


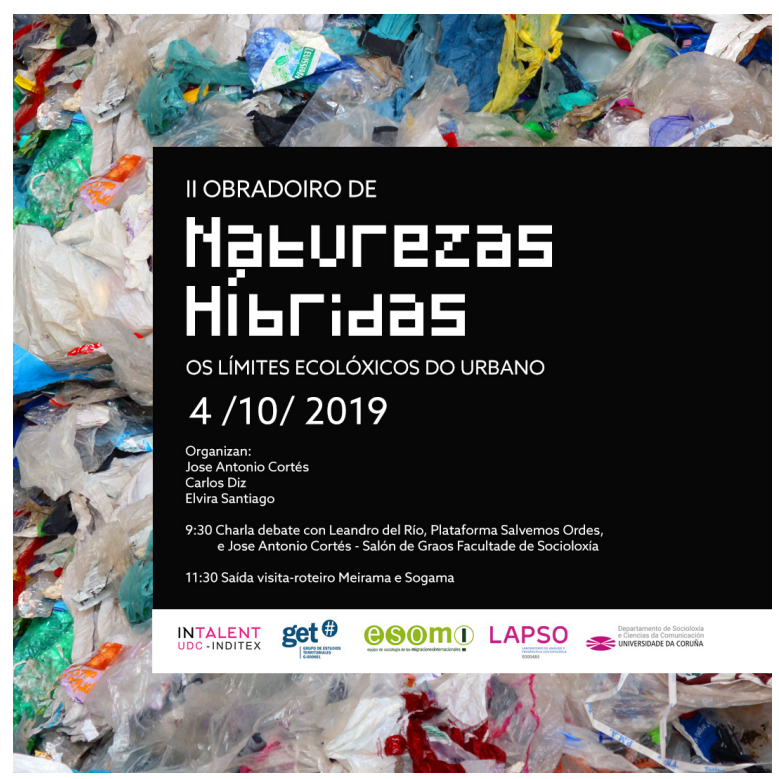

Imagen 1. Cartel informativo II Taller Naturalezas Híbridas

\section{RESULTADOS}

A través de la participación en el II Taller de Naturalezas Hibridas, el alumnado pudo problematizar y reflexionar sobre espacios más o menos invisibilizados en la cotidianidad de la vida en la ciudad, pero imprescindibles para la gestión de su suministro eléctrico y sus residuos. Esta reflexión supera la clásica división entre lo urbano y lo natural como dominios separados, situándonos ante entes híbridos, que retan las divisiones del conocimiento experto entre disciplinas aplicadas.

Metodológicamente, el taller propuso una triangulación en la que ensamblar la etnometodología, la etnografía y la acción participativa y que sirve de apoyo o complemento a los contenidos de las asignaturas optativas del Grado de Sociología: "Riesgo, Cultura y Medioambiente"; "Globalización, Medioambiente y Población"; y "Acción Colectiva y Cooperación Social", de las que son docentes los firmantes de este texto, investigadores especializados en el campo de la sociología, la antropología y las ciencias ambientales, y miembros de tres equipos de investigación de la facultad de sociología (el Grupo de Análisis y 
Prospectiva Sociopolítica, LAPSO; el Grupo de Estudios Territoriales, GET; y el Equipo de Sociología de las Migraciones Internacionales, ESOMI), una prueba más de hibridación disciplinar e innovación pedagógica.

Por último, para conocer la valoración del alumnado hacia la experiencia, se organizó un grupo de discusión en el que participaron seis estudiantes y en el que se abordaron cuatro bloques temáticos: Ios motivos que facilitaron la participación, la interpretación de los agentes y del territorio, la relación con la ciudad y propuestas para siguientes ediciones. El alumnado destacó que la confianza en las personas que organizaban la actividad fue el factor principal que condujo a su participación, a lo que le sigue el interés por participar en una experiencia transversal entre materias en la que participan personas expertas y activistas ajenas al ámbito académico. La participación en esta actividad ayudó a (re)conocer los límites ecológicos de lo urbano en el contexto metropolitano coruñés, a superar el pensamiento abstracto hacia las cuestiones relacionadas con la sostenibilidad ambiental y a enfrentar física y sensorialmente los impactos locales de la globalización.

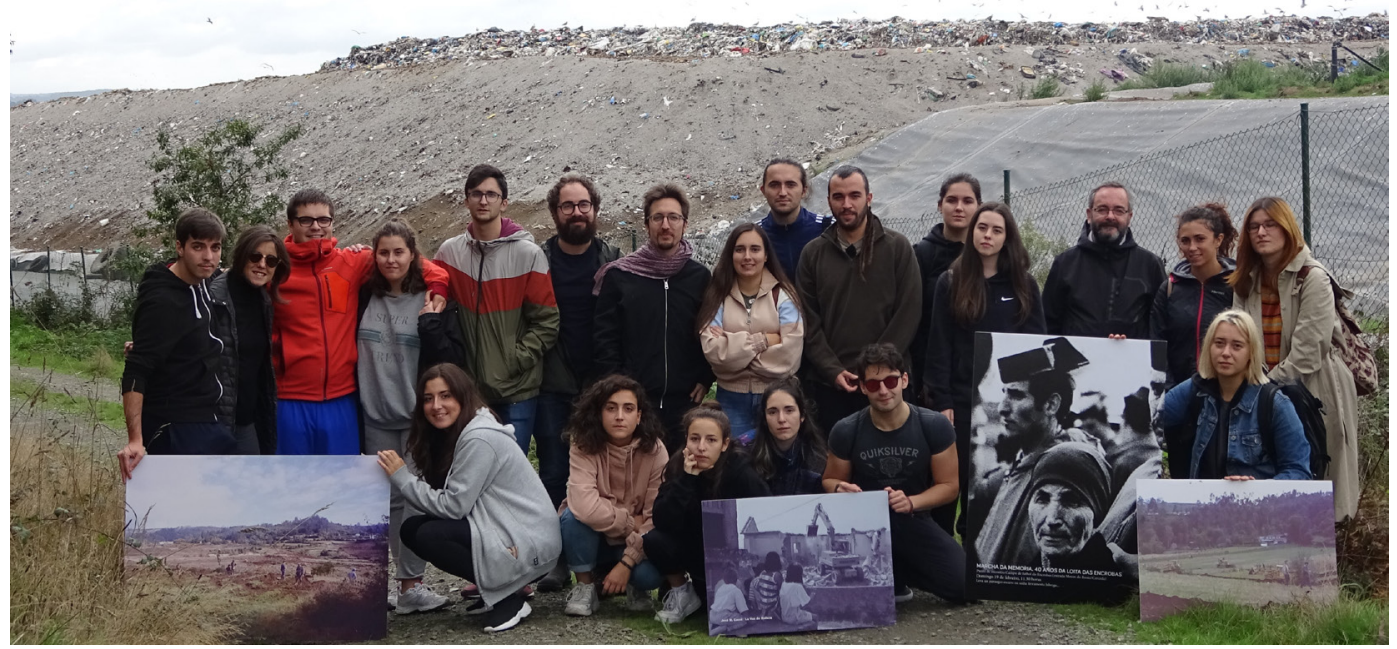

Imagen 2. Foto de grupo 


\section{CONCLUSIONES}

La buena acogida de esta segunda edición del Taller de Naturalezas Híbridas permite confiar en poder mantener la actividad en los próximos cursos abriéndola a la participación de estudiantes y profesorado de disciplinas afines. No obstante, la continuidad de la actividad dependerá de la capacidad para adaptar la organización a las exigencias del calendario académico y de encontrar estrategias que permitan introducir este tipo de prácticas de innovación docente en las metodologías de las materias, encontrando mecanismos que permitan una evaluación ajustada a las competencias adquiridas.

\section{REFERENCIAS}

Abadía, M., Mariano, J. \& Martín Vallespín, E. (2012). Universidad y desarrollo sostenible: análisis de la rendición de cuentas de las universidades públicas desde un enfoque de responsabilidad social. Revista Iberoamericana de Contabilidad de Gestión, 10 (19), pp. 1-18.

Diz, C., Santiago-Gómez, E. \& Cortes-Vazquez, Jose A. (2019). La co-producción de la Naturaleza Urbana. En De la Torre Fernández, E. (Ed.), Contextos universitarios transformadores: construíndo espazos de aprendizaxe (pp. 333-344). A Coruña: Cufie, Universidade da Coruña. A Coruña.

Lozano, R., Lukman, R., Lozano, F.J., Huising, D. \& Lambrechts, W. (2013). Declarations for sustainability in higher education: becoming better leaders, through addressing the universirty system. Journal of Cleaner Production, 48, pp. 10-49.

Savelyeva, T. y McKenna, J. (2011). Campus sustainability: emerging curricula models in higher education. International Journal of Sustainability in Higher Education, 12 (1), 55-66.

Vallaeys, F. (2006). Breve marco teórico de Responsabilidad Social Universitaria. Lima: Pontificia Universidad Católica del Perú. 
